\title{
REVIEW MANUSCRIPT
}

\section{Reliable and Cost-Effective Serodiagnosis of Rheumatoid Arthritis}

${ }^{1}$ Pieter W.A. Meyer, ${ }^{2}$ Mahmood M.T.M. Ally, ${ }^{3}$ Ronald Anderson.

${ }^{1}$ Department of Immunology, Faculty of Health Sciences, University of Pretoria and Tshwane Academic Division of the National Health Laboratory Service, Pretoria, South Africa, ${ }^{2}$ Department Internal Medicine, Faculty of Health Sciences, University of Pretoria, Pretoria, South Africa, ${ }^{3}$ Department of Immunology, Faculty of Health Sciences, University of Pretoria, Pretoria, South Africa.

Author e-mail addresses:

M Ally: $\quad$ mahmood.ally@up.ac.za

Prof R Anderson: $\quad \underline{\text { ronald.anderson@up.ac.za }}$

Corresponding Author: $\quad$ PWA Meyer

Adress: $\quad 5$ Bophelo Rd, Department Immunology, University of Pretoria, Prinshof Campus, South Africa.

E mail: $\quad$ pieter.meyer@up.ac.za

Telephone: $\quad$ +27 $\quad$ +

Fax: $\quad+27123230732$

Running title: Cost-effective laboratory testing for RA 


\section{Abstract}

Early diagnosis of patients with rheumatoid arthritis (RA) optimises therapeutic benefit and the probability of achieving disease remission. Notwithstanding clinical acumen, early diagnosis is dependent on access to reliable serodiagnostic procedures, as well as on the discerning application and interpretation of these. In the case of RA, however, no diseasespecific serodiagnostic procedure is available due to the multifactorial and polygenic nature of this autoimmune disorder. This has resulted in the development of an array of serodiagnostic procedures based on the detection of autoantibodies reactive with various putative autoantigens. Other procedures based on measurement of elevations in the concentrations of systemic biomarkers of inflammation, most commonly acute phase reactants and cytokines/chemokines, are used as objective indices of disease activity. Following a brief overview of RA research in African populations, the current review is focused on those autoantibodies/biomarkers, specifically rheumatoid factor, anti-citrullinated peptide antibodies, and C-reactive protein, which are currently recognised as being the most reliable and cost-effective with respect to disease prediction and diagnosis, as well as in monitoring activity and outcome.

Keywords: anti-citrullinated peptide antibodies; autoantibodies; C-reactive protein; inflammatory biomarkers; rheumatoid factor.

Abbreviations: ACPA, anti-citrullinated peptide antibodies; CRP, C-reactive protein; Ig, immunoglobulin; RF, rheumatoid factor; DAS, Disease Activity Score; SDAI, Simplified Disease Activity Index; DMARD, Disease-modifying antirheumatic drug. 


\section{Introduction}

Greater than $70 \%$ of poor people live in rural and remote regions of Africa[1], often with limited, if any, access to modern health care facilities and specialist clinicians. Consequently, it is highly probable that rheumatoid arthritis (RA), which has a prevalence of $1-3 \%$ in developed countries, is substantially under-diagnosed in our continent. Failure to diagnose RA at an early stage not only imposes considerable physical, emotional and socioeconomic hardship on patients, but also increases the demands on national health care budgets and facilities due to presentation of patients with late-stage, often therapy-refractory, disease. A twofold approach is necessary to address this problem. Firstly, ease of access of patients to specialist rheumatologists and appropriate therapies. Secondly, the availability of reliable, cost-effective serodiagnostic procedures, which is the major topic of this review, preceded by a brief historical overview of RA research in Africa.

The primary aims of this review are: i) to evaluate the utility of biomarkers published recently from data accrued from the African continent; ii) to assess the utility and cost-effectiveness of those biomarkers which improve the management of $R A$; and iii) to formulate recommendations in respect of optimising disease management in relation to laboratory testing.

\section{Rheumatoid arthritis research in Africa}

RA is a debilitating disease and has a severe impact on mobility and life expectancy of patients. This could be alleviated if patients were diagnosed early, enabling more accurate prediction of severe erosive disease, implementation of aggressive therapy and attainment of low disease activity. Early attainment of at least low disease activity, is not only beneficial to the patient, but also has significant cost-saving implications [2-5]. This has resulted in the search for reliable, affordable biomarkers with improved diagnostic and/or predictive potential. 
Multiple biomarkers of disease activity in RA have been evaluated in studies originating from both Africa [6-11] and elsewhere [12-16]. In addition, commercial ventures have also focused on developing multi-array test profiles that may improve cost-effective management of RA $[15,17]$. However, estimating the cost of RA disease burden in the South African and continental African setting in general is lacking, with the majority of articles relating to this topic having been published prior to 1990[18]. Exceptions include a 2009 study originating from Kenya which concluded that the majority of RA patients present at a relatively young age and are sub-optimally treated, which impacts negatively on disease control and quality of life [19], while a 2010 article highlighted the prevalence and functional impact of musculoskeletal conditions, including RA, in a small cohort in Cape Town [20]. Consequently, information is either wholly out-dated or restricted to limited geographical regions.

In a systematic review published in 2012 by Dowman et al., assessing the burden of RA in Africa, the authors commented on the limited number of publications emanating from the continent. Of the initial 80 publications identified, only 10 reported on the prevalence of RA and these were limited to South Africa, Nigeria and Uganda. The authors concluded that the information was inadequate to guide national health policies with respect to RA [18]. With respect to innovations in diagnosis, a study from Cameroon reported that measurement of ACPA (anti-citrullinated protein antibodies) was useful as a biomarker of RA and that the HLA-DRB1 shared epitope (SE), a genetic marker of disease susceptibility, was present in their RA population, but in lower frequency than in Caucasians. SE was also not a predictor of the presence of RF (rheumatoid factor) IgM (immunoglobulin M), RF IgA (immunoglobulin A) or ACPA using either of the $2^{\text {nd }}$ or $3^{\text {rd }}$ generation anti-CCP (cyclic citrullinated peptide) antibody tests [21].

A recent study from South Africa has focused on identifying immunodiagnostic biomarkers, which if used in combination with clinical disease activity indices and socio-demographic 
features of the patients, optimise the management and outcome of newly-diagnosed, DMARD-naïve RA patients with early disease.

In summary, the findings reported were as follows:

- RF, like ACPA, is significantly associated with erosive disease, smoking and functional disability. Combination testing of RF and ACPA had the best specificity and positive predictive value [22]

- Systemic pro-inflammatory and anti-inflammatory cytokines were elevated in DMARD-naïve RA patients, some of which were strongly and positively correlated with RF, ACPA, and acute phase reactants, as well as with HDA (high disease activity) [6]

- Patients unresponsive to DMARDs after 6 months of treatment are unlikely to improve at 12 months of treatment, underscoring the need for better disease management to attain LDA (low disease activity) or remission earlier in disease [23]. A large proportion of indigenous South Africans suffering from RA still has substantial functional disability and suboptimal mental health despite being on DMARD therapy for 12 months. A need to further investigate interventions in support programmes and biological therapies in this population was identified [24]

- The HLA-DRB1 SE has significant expression in the Black South African RA population. Classification according to the Du Montcel's revised criteria [25] indicated an overexpression of high-risk alleles associated with elevated clinical and biomarkers linked with disease severity [7]

- Rheumatoid nodules (RN), which were identified in $22.8 \%$ of the RA cohort, were significantly associated with HDA, SJC (swollen joint counts) and radiographic scores, as well as with certain cytokines, but not RF and ACPA [24]

- After 6 months of therapy SDAI, ACPA and certain circulating cytokines were dramatically reduced in the cohort, indicating possible prognostic value of a select group of circulating cytokines when used in conjunction with ACPA.[11] 


\section{Rheumatoid Factor (RF) and/or anti-Citrullinated Peptide antibodies (ACPA):}

The 2010 Rheumatoid Arthritis Classification Criteria, an ACR/EULAR (American College of Rheumatology/European League against Rheumatism) collaborative initiative, has incorporated rheumatoid factor (RF) and/or ACPA (anti-citrullinated protein antibody) serological tests, for the first time, of which a score is assigned according to the positivity and level of either RF and/or ACPA results, which forms part of the scoring to contribute to the overall likelihood of diagnosing of RA [26].

RF was the first autoantibody described that was associated with RA. Scientists discovered that sera from RA patients agglutinated SRBC (Sheep Red Blood Cells) that were sensitised by rabbit antibodies. In 1940, the term "rheumatoid factor" was used when the correlation between the agglutination factor and RA was confirmed. RF antibodies are predominantly $\lg M(R F-\lg M)$, but may also be of the $\lg G$ and $\lg A$ classes. RF targets the Fc region of human IgG In the $\gamma 2-\gamma 3$ cleft. The resultant immune complexes contribute to the pathogenesis of RA via complement activation.[27]

The most common methods in use for RF detection are: i) ELISA (Enzyme-Linked Immunosorbent Assay); ii) indirect agglutination using latex, charcoal or human RBC (Red Blood Cells) as carrier particles loaded with IgG of human or rabbit origin; and iii) laser or rate nephelometry in which latex is coated with $\lg G$ (human) with the amount of light scatter caused by the RF IgG-coated latex bound by the RF IgM directly related to the concentration of RF.[28]

Although $\mathrm{RF}$ is present in a high percentage (>86\%) of RA patients, it is also present in other autoimmune diseases and infectious diseases, as well as in about $3-5 \%$ of the healthy population, albeit at lower concentrations $(<50 \mathrm{IU} / \mathrm{ml})[29,30]$ Transient increases in RF IgM are also part of the normal immune response and are detectable during infection in healthy individuals and especially in the elderly [31]. However, the persistence of RF in the setting of 
titres in excess of 50 or $100 \mathrm{IU} / \mathrm{ml}$ improves the specificity for $\mathrm{RA}$ to $\geq 88 \%$ and $\geq 95 \%$, respectively [32].

It has also been reported that RF $\lg M$ and $R F \lg A$ in combination are almost exclusively found in RA patients and that distinguishing between RF single isotypes is more specific and sensitive than other detection methods [33].

Classically, RF has been the most useful biomarker in the diagnosis of RA until the advent of ACPA [34]. Citrullination or deimination is the term used for the post-translational modification of the amino acid arginine in a protein via conversion to the amino acid citrulline. This reaction is mediated by enzymes known as peptidylarginine deiminases (PADs). The conversion of arginine to citrulline has significant consequences for the structure and function of proteins, since arginine is positively charged at neutral $\mathrm{pH}$, whereas citrulline is uncharged. This increases the hydrophobicity of the protein, leading to changes in protein dimensional orientation [35].

Schellekens et al. described the existence of autoantibodies to filaggrin subunits which contained high numbers of arginine residues which were modified to citrulline by peptidylarginine deiminase in vitro. They also showed that these antibodies reacted with anti-perinuclear factor/anti-keratin (APA/AKA) and demonstrated a strong correlation with the citrulline peptides, confirming that the citrullinated peptides represent the major epitopes recognised by APA/AKA as seen by indirect immunofluorescence (IIF) using bucal mucosa cells [34].

Van Boekel et al. first demonstrated that ACPA were detected early in disease and predicted erosive disease.[29] In 2005 Rantapää-Dahlqvist concluded that measurement of ACPA is superior to RF in the early diagnosis of RA and is also a better indicator of disease severity.[36] The diagnostic and prognostic utility of measurement of ACPA was conclusively established by van Boekel et al. in 2007 who established the specificity of ACPA for RA. 
Recently, it was reported that ACPA titres decreased significantly after treatment with DMARDs in a therapy-naïve cohort of black South Africans diagnosed with RA and that the ACPA levels were statistically significantly higher in those positive for the high-risk HLAalleles associated with RA.[11]

Following confirmation of the association of ACPA with RA, a major review by Aggerwal et al. (2008) which was focused on the application of ACPA as a diagnostic tool in respect of performance, precision correlation, cost-effectiveness and accuracy led to the following conclusions:

- "ACPA assays have good predictive value because they are associated with the known genetic and epidemiologic risk factors for RA, and also identify a population of RA patients with more severe, erosive joint disease, who are at high risk for more rapid joint destruction

- $\mathrm{RF}$ and ACPA are two different autoantibody systems and do not measure or reflect the same underlying biology

- ACPA assays are becoming increasingly available and less expensive. Costeffectiveness analyses suggest that testing of ACPA in patients presenting with undifferentiated arthritis is cost-effective, particularly in relation to eliminating the indirect costs associated with delayed diagnosis

- ACPA offers similar sensitivity, but higher specificity, for RA than RF in early disease. When used in the identification of patients with sub-clinical RA, as well as those presenting with early undifferentiated symptoms, a high risk population, the positive predictive value of the ACPA assay is in the order of $95 \%$.

- In the setting of a relatively high clinical suspicion (pre-test probability) and a positive ACPA result, the patient has a high likelihood of having or developing RA. If ACPA is negative, further testing may be indicated depending on the level of clinical suspicion."[37] 
The combination of testing for both ACPA and RF to obtain a sensitivity $>99 \%$ is well known [38] and is reinforced by a study undertaken by Infantino et al. which concluded that a combination of ACPA and RF $\lg M$ and $\lg A$ isotype testing delivers the best screening procedure for detection of early RA [39].

\section{Other putative autoantigens associated with $R A$}

Notwithstanding RF and ACPA, RA is associated with the presence of several other autoantibodies directed against different antigenic determinants.

Although measurement of anti-Sa (citrullinated vimentin), and nuclear ribonucleoprotein A2 and its splice variant B1 (hnRNP A2B1), also known as anti-RA33, as well as anti-MCV (Mutated Citrullinated Vimentin) has been reported, their diagnostic utilities are significantly less than those of RF and ACPA. Anti-Sa has been shown to be associated with higher RF and ACPA values, as well as with HDA, but its overall sensitivity of is lacking in comparison with RF and ACPA. Citrullinated vimentin has been detected in the synovium, consistent with a role for this putative autoantigen in the pathogenesis of RA [40, 41].

Circulating antibodies against anti-RA33, have also been reported to be present in SLE (systemic lupus erythematosus), MCTD (mixed connective tissue disease) and RA, and to be specific for the RNA binding domains of hnRNP-A2; however, as mentioned above, the overall sensitivity and specificity for RA is low(20-40\%) [42].

Anti-MCV antibodies, although comparable to anti-CCP in specificity, lack prognostic value and have not gained significant traction to replace ACPA. Measurement of anti-MCV antibodies is, however, suggested as an alternative in the setting of negative results for RF and ACPA and there is a strong clinical indication of RA [43]. In addition, these have varied sensitivity and prognostic values as summarised in Table1.

Anti-GPI(glucose-6-phosphate isomerase), -collagen, -BiPp68(stress protein), -hsp90(heat shock protein 90), -calpastatin, -a-enolase, -PAD4 (peptidylarginine deiminase, type IV) 
antibodies have not demonstrated meaningful diagnostic or prognostic sensitivity or specificity for RA (also show in Table 1).

Table 1. Putative autoantigens present in RA

\begin{tabular}{|c|c|c|c|}
\hline Antigen & Antibody & $\begin{array}{l}\text { Sensitivity } \\
(\%)\end{array}$ & $\begin{array}{l}\text { Specificity } \\
(\%)\end{array}$ \\
\hline Citrullinated proteins & $\begin{array}{l}\text { Anti-fibrin, anti-filaggrin, anti- } \\
\text { vimentin (MCV) }\end{array}$ & $50-91$ & $>97^{a}$ \\
\hline $\begin{array}{l}\text { Heterogeneous nuclear } \\
\text { ribonucleoprotein A2B1 }\end{array}$ & Anti-RA33 & $\leq 36$ & NRADA \\
\hline Collagen II & Anti-collagen & $\leq 30$ & NRADA \\
\hline Stress proteins & Anti-BiPp68, anti-hsp90 & $\leq 68$ & $71-99^{a}$ \\
\hline Calpastatin & Anti-calpastatin & $24-57(83)^{a}$ & $\begin{array}{l}\text { Not verified } \\
(96)^{\mathrm{a}}\end{array}$ \\
\hline Citrullinated vimentin & Anti-Sa & $\leq 40$ & $85-95$ \\
\hline a-Enolase (citrullinated) & Anti- $\alpha$-enolase & 46 & $a^{a}$ \\
\hline $\begin{array}{l}\text { Glucose-6 phosphate isomerase } \\
\text { (GPI) }\end{array}$ & Anti-GPI & $\leq 64$ & $>95^{\mathrm{a}}$ \\
\hline PAD4 & Anti-PAD4 & $36-42^{a}$ & $\mathrm{a}^{\mathrm{a}}$ \\
\hline
\end{tabular}

$M C V$ mutated citrullinated vimentin, NRADA non-RA differentiated arthritis

Compiled from Refs. $[\underline{29}, \underline{32}, \underline{36}, \underline{49}, \underline{50}]$

${ }^{\text {a }}$ Requires confirmation

\section{C-reactive protein}

C-reactive protein is an integral component of RA disease activity scores (Disease Activity Score - DAS28-CRP and SDAI - Simplified Disease Activity Index) and multi-biomarker disease activity algorithms, individually, or in conjunction with RF, ACPA and gender. Furthermore, persistently high CRP values in RA patients are associated with greater risk for progressive joint deterioration and are indicative of the need for more aggressive treatment, whereas a decrease in CRP values is indicative of an effective treatment regimen.[44]

A retrospective study utilising sera from blood donors who were later diagnosed with RA demonstrated that elevated levels of CRP are evident several years before the appearance of clinical manifestations of disease activity and that these were independent of the presence of other RA-associated biomarkers.[45] It has been reported that CRP, in conjunction with ACPA, but not RF, remains elevated post -DMARDs treatment, especially in patients treated 


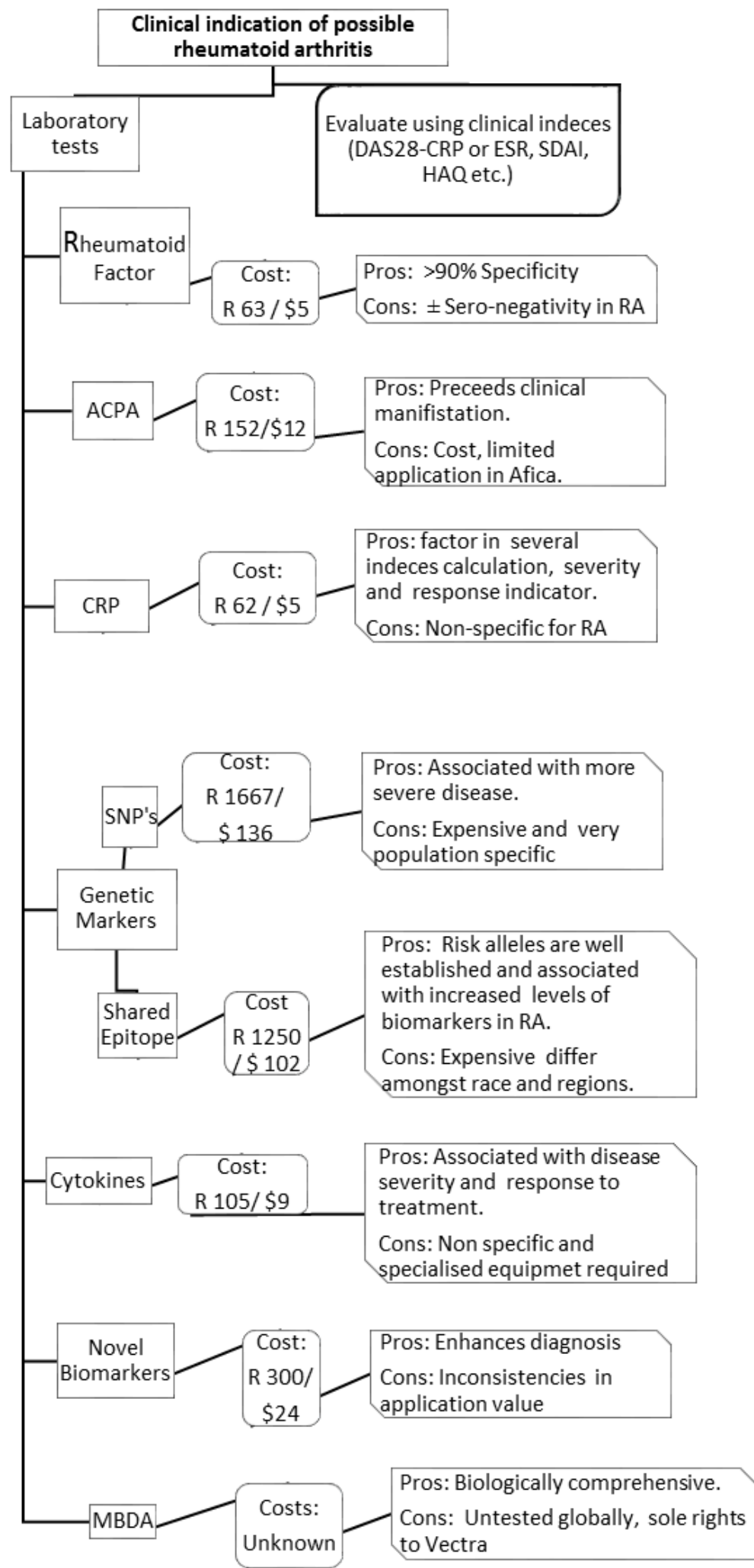

Figure 1. Laboratory tests available for diagnosis of RA and assessment of disease severity, together with their costs and advantages (Pros) and disadvantages (Cons).

Key: DAS (Disease Activity Score) 28, CRP (C-Reactive Protein), ESR (Estimated Sedimentation Rate), SDAI (Simplified Disease Activity Score), HAQ (Health Assessment Questionnaire), ACPA (Cyclic Citrullinated Peptide), SNP (Short Nucleotide Polymorphism), MBDA (Multibiomarker Disease Activity). 
with combination therapy (methotrexate (MTX) and corticosteroids) when compared with patients on MTX monotherapy, supporting the contention that measurement of CRP in combination with ACPA has both diagnostic and prognostic potential in RA.[11]

Measurement of CRP in combination with RF, ACPA and HLA-DRB1-associated genes has also been shown to predict radiographic progression and improve the management of RA [46-48].

\section{Recommendations}

Only limited budgets are often available to health providers in developing countries and often compete with priority programmes such as HIV and TB to curb high burdens of disease. Non-communicable diseases such as RA may therefore receive a lower than necessary priority requiring discerning choice of the most reliable and cost-effective laboratory diagnostic procedures. Those currently available procedures which have both diagnostic and prognostic potential are shown in Figure 1, together with their associated costs, advantages and limitations.

Figure 2 indicates those biomarkers and their associated relevance to RA which can be tested for at different stages of disease progression. The flow begins with genetic markers to determine the possible predisposition of developing RA before clinical manifestation, together with possible environmental triggers. This is followed by detection of those biomarkers incorporated into the ACR/EULAR 2010 classification. These assist in both diagnosis and post-classification monitoring.

Realistically however, RF and ACPA are the current cornerstones of RA diagnosis and when used in combination ensure the highest sensitivity and PPV. Other circulating biomarkers such as CRP in particular, and possibly certain cytokines when used in combination with RF and ACPA, may contribute to assessment of disease severity and outcome. 


\section{Genetic Risk}

- Shared epitope, SNP

- Population dependant.

- Risk allele = HDA.

- Environmental factors

- Smoking status, pollution.

- Smokers - poor responders - greater risk of co-morbidity.

\section{Post Diagnosis of RA}

- RF, ACPA, CRP monitoring

- $\quad \mathrm{RF}+\mathrm{ACPA}$ increased $\rightarrow$ 个morbidity.

- Unchanged ACPA, CRP levels posttreatment $=$ non-responders.

- Circulating Cytokines

- Response to DMARDs, biological therapies.

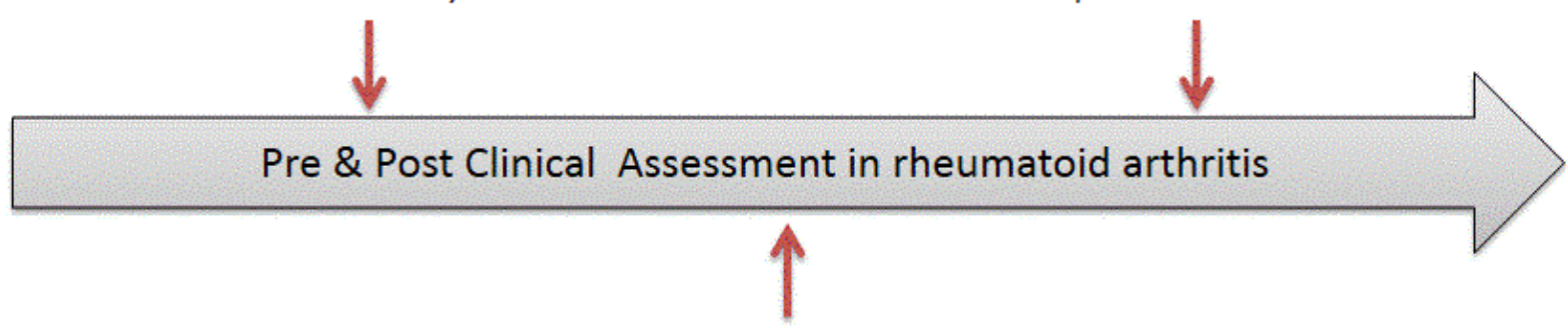

\section{Pre-Clinical Autoimmunity}

- RF, ACPA, CRP

- Present in elevated levels.

- $\mathrm{RF}>1.5$ years before clinical onset.

- ACPA $>4.5$ years before clinical onset.

- $\quad$ CRP $>1$ year before clinical onset.

Figure 2. The first phase of assessment is to establish the possible risk factors and associated environmental triggers which may predispose for development of RA together with measurement of those autoantibodies the appearance of which precedes and is predictive disease onset. Ultimately, the preceding events may lead to the manifestation of inflammatory RA. Highlighted in each phase are the possible laboratory tests available and their added value.

Given the ongoing severe financial constraints confronting national health budgets of developing countries, measurement of RF or ACPA on presentation is essential, preferably in combination, or, alternatively, RF only followed by ACPA according to clinical intuition.

\section{References}

[1]. Ifad. Rural poverty report 2011 [Electronic Report] Rome, Italy: www.ifad.org; 2011 [cited 201513 July]. Available from: http://www.ifad.org/rpr2011/report/e/overview.pdf.

[2]. Pugner KM, Scott DI, Holmes JW, Hieke K. The costs of rheumatoid arthritis: an international long-term view. Seminars in Arthritis and Rheumatism. 2000;29(5):305-320.

[3]. Behzad H. Rheumatoid Arthritis: Early diagnosis and treatment outcomes. Caspian Journal of Internal Medicine. 2011;2(1):161-170. 
[4]. Radner H, Smolen JS, Aletaha D. Remission in rheumatoid arthritis: benefit over low disease activity in patient-reported outcomes and costs. Arthritis Research \& Therapy. 2014;16(1):R56.

[5]. Smolen JS, Breedveld FC, Burmester GR, Bykerk V, Dougados M, Emery P, et al. Treating rheumatoid arthritis to target: 2014 update of the recommendations of an international task force. Annals of Rheumatic Diseases. 2015;0:1-13.

[6]. Meyer PWA, Hodkinson B, Mahmood A, Musenge E, Wadee AA, Fickl H, et al. Circulating cytokine profiles and their relationship with autoantibodies, acute phase reactants, and disease activity in patients with rheumatoid arthritis. Mediators Of Inflammation. 2010;2010/158154.

[7]. Meyer PW, Hodkinson B, Ally M, Musenge E, Wadee AA, Fickl H, et al. HLA-DRB1 shared epitope genotyping using the revised classification and its association with circulating autoantibodies, acute phase reactants, cytokines and clinical indices of disease activity in a cohort of South African rheumatoid arthritis patients. Arthritis Research \& Therapy. 2011;13(5):R160.

[8]. Ally MM, Hodkinson B, Meyer PW, Musenge E, Tikly M, Anderson R. Serum matrix metalloproteinase-3 in comparison with acute phase proteins as a marker of disease activity and radiographic damage in early rheumatoid arthritis. Mediators Of Inflammation. 2013;2013:183653.

[9]. May A, Hazelhurst S, Li Y, Norris SA, Govind N, Tikly M, et al. Genetic diversity in black South Africans from Soweto. BMC Genomics. 2013;14(1):1-12.

[10]. Govind N, Choudhury A, Hodkinson B, Ickinger C, Frost J, Lee A, et al. Immunochip Identifies Novel, and Replicates Known, Genetic Risk Loci for Rheumatoid Arthritis in Black South Africans. Molecular Medicine. $2014 ; 20(1): 341-349$.

[11]. Ally MM, Hodkinson B, Meyer PW, Musenge E, Tintinger GR, Tikly M, et al. Circulating anti-citrullinated peptide antibodies, cytokines and genotype as biomarkers of response to disease-modifying antirheumatic drug therapy in early rheumatoid arthritis. BMC Musculoskeletal Disorders. 2015;16:130.

[12]. Nam J, Villeneuve E, Emery P. The role of biomarkers in the management of patients with rheumatoid arthritis. Current rheumatology reports. 2009;11(5):371-377.

[13]. Curtis JR, Van Der Helm-Van Mil AH, Knevel R, Huizinga TW, Haney DJ, Shen Y, et al. Validation of a novel multibiomarker test to assess rheumatoid arthritis disease activity. Arthritis care \& research. 2012;64(12):1794-1803.

[14]. Trouw LA, Mahler M. Closing the serological gap: promising novel biomarkers for the early diagnosis of rheumatoid arthritis. Autoimmunity Reviews. 2012;12(2):318-322.

[15]. Centola M, Cavet G, Shen Y, Ramanujan S, Knowlton N, Swan KA, et al. Development of a MultiBiomarker Disease Activity Test for Rheumatoid Arthritis. PLoS ONE. 2013;8(4):e60635.

[16]. Burska A, Boissinot M, Ponchel F. Cytokines as Biomarkers in Rheumatoid Arthritis. Mediators Of Inflammation. 2014;2014:24. 
[17]. Michaud K, Strand V, Shadick NA, Degtiar I, Ford K, Michalopoulos SN, et al. Outcomes and costs of incorporating a multibiomarker disease activity test in the management of patients with rheumatoid arthritis. Rheumatology (Oxford). 2015;54(9):1640-1649.

[18]. Dowman B, Campbell RM, Zgaga L, Adeloye D, Chan KY. Estimating the burden of rheumatoid arthritis in Africa: A systematic analysis. Journal of Global Health. 2012;2(2):020406.

[19]. Owino BO, Oyoo GO, Otieno CF. Socio-demographic and clinical aspects of rheumatoid arthritis. East African Medical Journal. 2009;86(5):204-211.

[20]. Parker R, Jelsma J. The prevalence and functional impact of musculoskeletal conditions amongst clients of a primary health care facility in an under-resourced area of Cape Town. BMC Musculoskeletal Disorders. 2010;11:2-2.

[21]. Singwe-Ngandeu M, Finckh A, Bas S, Tiercy JM, Gabay C. Diagnostic value of anti-cyclic citrullinated peptides and association with HLA-DRB1 shared epitope alleles in African rheumatoid arthritis patients. Arthritis Research \& Therapy. 2010;12(2):R36.

[22]. Hodkinson B, Meyer PW, Musenge E, Ally MM, Wadee AA, Anderson R, et al. The diagnostic utility of the anti-CCP antibody test is no better than rheumatoid factor in South Africans with early rheumatoid arthritis. Clinical rheumatology. 2010;29(6):615-618.

[23]. Hodkinson B, Musenge E, Ally M, Meyer PW, Anderson R, Tikly M. Response to traditional diseasemodifying anti-rheumatic drugs in indigent South Africans with early rheumatoid arthritis. Clinical rheumatology. 2011;31:613-619.

[24]. Hodkinson B, Meyer PW, Musenge E, Ally M, Anderson R, Tikly M. Exaggerated circulating Th-1 cytokine response in early rheumatoid arthritis patients with nodules. Cytokine. 2012;60(2):561-564.

[25]. Tezenas Du Montcel S, Michou L, Petit-Teixeira E, Osorio J, Lemaire I, Lasbleiz S, et al. New classification of HLA-DRB1 alleles supports the shared epitope hypothesis of rheumatoid arthritis susceptibility. Arthritis and Rheumatism. 2005;52(4):1063-1068.

[26]. Aletaha D, Neogi T, Silman AJ, Funovits J, Felson DT, Bingham CO, 3rd, et al. 2010 Rheumatoid arthritis classification criteria: an American College of Rheumatology/European League Against Rheumatism collaborative initiative. Arthritis and Rheumatism. 2010;62(9):2569-2581.

[27]. Renaudineau Y, Jamin C, Saraux A, Youinou P. Rheumatoid factor on a daily basis. Autoimmunity. $2005 ; 38(1): 11-16$.

[28]. Food US, Drug A. Review Criteria For Assessment Of Rheumatoid Factor (Rf) In Vitro Diagnostic Devices Using Enzyme-Linked Immunoassay (Eia), Enzyme Linked Immunosorbent Assay (Elisa), Particle Agglutination Tests, And Laser And Rate Nephelometry. FDA; 1997.

[29]. Van Boekel MA, Vossenaar ER, Van Den Hoogen FH, Van Venrooij WJ. Autoantibody systems in rheumatoid arthritis: specificity, sensitivity and diagnostic value. Arthritis Research. 2002;4(2):87-93. 
[30]. Ingegnoli F, Castelli R, Gualtierotti R. Rheumatoid Factors: Clinical Applications. Disease Markers. 2013;35(6):727-734.

[31]. Valesini G, Alessandri C. Anticitrullinated protein/peptide antibodies and rheumatoid factors: two distinct autoantibody systems. Arthritis Research \& Therapy. 2009;11(5):125 (doi:110.1186/ar2786).

[32]. Steiner G, Smolen JS. Autoantibodies in rheumatoid arthritis and their clinical significance. Arthritis Research. 2002;4 Suppl 2:S1-5.

[33]. Jonsson T, Steinsson K, Jonsson H, Geirsson AJ, Thorsteinsson J, Valdimarsson H. Combined elevation of $\lg \mathrm{M}$ and $\lg \mathrm{A}$ rheumatoid factor has high diagnostic specificity for rheumatoid arthritis. Rheumatology International. 1998;18(3):119-122.

[34]. Schellekens GA, Visser H, De Jong BaW, Van Den Hoogen FHJ, Hazes JMW, Breedveld FC, et al. The diagnostic properties of rheumatoid arthritis antibodies recognizing a cyclic citrullinated peptide. ARTHRITIS \& RHEUMATISM. 2000;43(1):155-163.

[35]. Vossenaar ER, Zendman AJ, Van Venrooij WJ, Pruijn GJ. PAD, a growing family of citrullinating enzymes: genes, features and involvement in disease. BioEssays :News and Reviews in Molecular, Cellular and Developmental Biology. 2003;25(11):1106-1118.

[36]. Rantapää-Dahlqvist S. Diagnostic and prognostic significance of autoantibodies in early rheumatoid arthritis. Scandinavian Journal of Rheumatology. 2005;24:83-96.

[37]. Aggarwal R, Liao K, Nair R, Ringold S, Costenbader KH. Anti-Citrullinated Peptide Antibody (ACPA) Assays and their Role in the Diagnosis of Rheumatoid Arthritis. Arthritis and Rheumatism. 2009;61(11):14721483.

[38]. Rantapää-Dahlqvist S, Boman K, Tarkowski A, Hallmans G. Up regulation of monocyte chemoattractant protein-1 expression in anti-citrulline antibody and immunoglobulin $M$ rheumatoid factor positive subjects precedes onset of inflammatory response and development of overt rheumatoid arthritis. Annals of the Rheumatic Diseases. 2007;66(1):121-123.

[39]. Infantino M, Manfredi M, Meacci F, Sarzi-Puttini P, Ricci C, Atzeni F, et al. Anti-citrullinated peptide antibodies and rheumatoid factor isotypes in the diagnosis of rheumatoid arthritis: an assessment of combined tests. Clinica Chimica Acta. 2014;436:237-242.

[40]. Hou YF, Sun GZ, Sun HS, Pan WP, Liu WB, Zhang CQ. Diagnostic value of anti-Sa and anticitrullinated protein antibodies in rheumatoid arthritis. Journal of Rheumatology. 2012;39(8):1506-1508.

[41]. El-Gabalawy HS, Wilkins JA. Anti-Sa antibodies: prognostic and pathogenetic significance to rheumatoid arthritis. Arthritis Research \& Therapy. 2004;6(2):86-89.

[42]. Skriner K, Sommergruber WH, Tremmel V, Fischer I, Barta A, Smolen JS, et al. Anti-A2/RA33 autoantibodies are directed to the RNA binding region of the A2 protein of the heterogeneous nuclear 
ribonucleoprotein complex - Differential epitope recognition in rheumatoid arthritis, systemic lupus erythematosus, and mixed connective tissue disease. Journal of Clinical Investigation. 1997;100(1):127-135.

[43]. Luime JJ, Colin EM, Hazes JMW, Lubberts E. Does anti-mutated citrullinated vimentin have additional value as a serological marker in the diagnostic and prognostic investigation of patients with rheumatoid arthritis? A systematic review. Annals of the Rheumatic Diseases. 2010;69(2):337-344.

[44]. Otterness IG. The value of C-reactive protein measurement in rheumatoid arthritis. Seminars in Arthritis and Rheumatism. 1994;24(2):91-104.

[45]. Nielen MMJ, Van Schaardenburg D, Reesink HW, Twisk JWR, Van De Stadt RJ, Van Der Horst-Bruinsma $\mathrm{IE}$, et al. Increased levels of C-reactive protein in serum from blood donors before the onset of rheumatoid arthritis. ARTHRITIS \& RHEUMATISM. 2004;50(8):2423-2427.

[46]. Listing J, Rau R, Muller B, Alten R, Czerwony G, Gromnica-Ihle E. HLA-DRB1 genes, rheumatoic factor, and elevated C-reactive protein: Independent risk factors of radiographic progression in early rheumatoid arthritis. Journal of Rheumatology. 2000;27(9):2100-2109.

[47]. Yamane T, Hashiramoto A, Tanaka Y, Tsumiyama K, Miura Y, Shiozawa K, et al. Easy and accurate diagnosis of rheumatoid arthritis using anti-cyclic citrullinated peptide 2 antibody, swollen joint count, and Creactive protein/rheumatoid factor. Journal of Rheumatology. 2008;35(3):414-420.

[48]. Taylor P, Gartemann J, Hsieh J, Creeden J. A Systematic Review of Serum Biomarkers Anti-Cyclic Citrullinated Peptide and Rheumatoid Factor as Tests for Rheumatoid Arthritis. Autoimmune Dis. 2011;2011. 